\title{
ON SOLUTIONS OF DIFFERENTIAL EQUATIONS WHICH POSSESS AN OSCILLATOIN THEOREM *
}

\section{HELEN A. MERRILL}

\section{§1. InTRODUCTION.}

The object of this paper is the study of differential equations possessing certain properties, later defined, which may be called Sturmian properties, since they characterize, equations first discussed by STURM in his celebrated Mémoire sur les équations linéaires $d u$ second ordre, in the first volume of Liou ville's Journal. This memoir of Sturm's is chiefly devoted to the study of the equation

$$
\frac{d}{d x}\left[K(x, \lambda) \frac{d y(x, \lambda)}{d x}\right]+G(x, \lambda) y(x, \lambda)=0,
$$

with a few slightly more general forms. At the close of the article he refers briefly to the fact that these results were first obtained by the use of equations in finite differences of the second order, but none of this work was ever published, $\dagger$ owing probably to the fact that at that time it was impossible to treat the subject with sufficient rigor by this method. In the present state of analysis STURM's original method may easily be placed on a satisfactory basis as regards rigor, and it is found to possess certain marked advantages, due to the ease with which recurrent relations satisfying the necessary conditions are set up, and to the possibility of generalizing much of the work. STURM's famous theorem in regard to the location of the real roots of an equation is only a result of the application of this general method to polynomials.

In order to establish on a rigorous basis the method of finite differences, use will be made of a recent theorem $\ddagger$ of Painleve and Picard in regard to the CAUChY-Lipschitz proof of the existence of a solution of a differential equation.

* Presented to the Society at the Boston meeting August 31-September 1, 1903. Received for publication August 10, 1903.

† A partial reconstruction of the original method of STURM, with proofs of several of the Sturmian theorems, was published in the Annals of Mathematics, January, 1902, by M. B. PORTER.

$\ddagger$ This theorem has reference to the uniformity of the convergence of the CAUCHY algorithm and to the interval of the convergence of the resulting solution. See p. 432. 
After recalling some familiar definitions and theorems of which use will be made, a method will be developed by which recurrent relations of the required character are constructed. Some properties of the functions which satisfy these relations will then be established, and it will be shown that these same properties characterize the differential equations, to which under certain conditions the recurrent relations lead.

\section{§ 2. Preliminary Definitions and Statements.}

Sturmían Sequence. A sequence of functions, $y_{0}(\lambda), y_{1}(\lambda) \cdots y_{n}(\lambda)$, may be called Sturmian when the number of real roots of $y_{n}(\lambda)$ lying between any two real values of the argument $\lambda$ is a function of the number of variations in the signs of the sequence lost or gained as $\lambda$ increases between these two values.

The functional relation here employed is that in general use, viz., the number of real roots between any two real values of $\lambda$ is equal to the difference between the number of variations of the sequence for these two values of $\lambda$. In order that a sequence may be Sturmian in this special sense the following conditions are sufficient:

I. 1. $y_{0}(\lambda)$ does not vanish throughout the interval considered.

2. Two consecutive functions cannot vanish for the same value of $\lambda$.

3. When an intermediate function vanishes, the two adjacent functions have unlike signs.

II. $y_{n}(\lambda)$ and $y_{n-1}(\lambda)$ have unlike signs just before $y_{n}(\lambda)$ vanishes, and like signs just after. A sufficient condition to secure this property is the following :

$$
\frac{d}{d \lambda}\left[\frac{y_{n}(\lambda)}{y_{n-1}(\lambda)}\right]_{y_{n}(\lambda)=0}>0
$$

REMARK. In case the functions have no derivatives, let it be possible to write $y_{n}(\lambda)$ in the vicinity of the point $a$, at which it vanishes, in the form

$$
y_{n}(\lambda)=(\lambda-a) T(\lambda),
$$

where $T(\lambda)$ is continuous and does not vanish for $\lambda=a$. We then have

$$
\frac{y_{n}(\lambda)}{y_{n-1}(\lambda)}=(\lambda-a) \frac{T(\lambda)}{y_{n-1}(\lambda)}
$$

an expression which must be finite for values of $\lambda$ in the vicinity of $a$, and vanish for $\lambda=a$. Condition II is then satisfied, provided in the vicinity of $a$,

$$
\frac{T(\lambda)}{y_{n-1}(\lambda)}>0 \text {. }
$$


The above conditions I and II will be referred to as the first and second Sturmian conditions respectively.

Character of the Functions Employed. In all this work it will be assumed that the functions under consideration, as well as the coefficients of the homogeneous recurrent relation which any three consecutive functions must satisfy, are real, single-valued, continuous, differentiable functions of a real parameter $\lambda$, which is supposed in every case to lie within a certain definite interval, $\lambda_{i} \leqq \lambda \leqq \lambda_{k}$. It is to be noted that none of the functions involved are necessarily analytic.

Notation. For convenience in writing, the argument of the functions will in general be omitted, but a consistent notation will be employed - the first letters of the alphabet denoting functions independent of $\lambda$, all other letters dẹnoting functions of $\lambda$.

\section{§3. Development of a Method of Forming Recurrent Relations such that the Functions Satisfying them form a Sturmian Sequence.}

Our first effort is to satisfy the second Sturmian condition, that is, to impose conditions sufficient to secure the increase of $y_{n} / y_{n-1}$ with $\lambda$ either throughout the interval considered or for values of $\lambda$ for which $y_{n}$ vanishes. It will appear later that condition I can then be readily imposed.

A homogeneous recurrent relation of the second order, $F\left(y_{m+1}, y_{m}, y_{m-1}\right)=0$, can be written in the form

$$
z_{m+1}=f\left(z_{m}\right) \quad(m=0,1,2 \cdots n-1) .
$$

where $z_{m+1}=y_{m+1} / y_{m}, z_{m}=y_{m} / y_{m-1}$. If now conditions be imposed sufficient to secure monotone increase of $z_{m+1}$ as $z_{m}$ increases monotone, the second Sturmian condition is evidently met. Our mode of procedure, briefly stated, is to selest a simple expression connecting $z_{m}$ and $z_{m+1}$, by which under certain conditions the second Sturmian condition is secured, and to operate upon it by linear transformations such that this condition is at every step conserved, while the first Sturmian condition also is ultimately satisfied. The simplest possible relation connecting $z_{m}$ and $z_{m+1}$ is of the linear fractional type:

$$
z_{m+1}=\frac{K_{m} z_{m}+L_{m}}{M_{m}^{2} z_{m}+N_{m}}
$$

which may be regarded as a transformation of the $z_{m}$ plane on the $z_{m+1}$ plane. Conditions are to be imposed upon $K_{m}, L_{m}, M_{m}, N_{m}$, sufficient to secure monotone increase of $z_{m+1}$ when $z_{m}$ increases monotone, both $z_{m+1}$ and $z_{m}$ having a range of values from $-\infty$ to $+\infty$. That is, as $z_{m}$ describes the real axis in the positive sense, $z_{m+1}$ is to describe the real axis in its plane, also in the positive sense. This transformation may be broken up into the following steps : 


$$
\begin{aligned}
z_{m}^{(1)} & =M_{m} z_{m}+N_{m} . \\
z_{m}^{(2)} & =\frac{1}{z_{m}^{(1)}} . \\
z_{m+1} & =\frac{L_{m} M_{m}-K_{m} N_{m}}{M_{m}} z_{m}^{(2)}+\frac{K_{m}}{M_{m}^{-}} .
\end{aligned}
$$

We shall first develop in detail the recurrent relation of which the differential equation treated by STURM may be regarded as the limiting form, and then indicate briefly how in the same way more general forms may be obtained.

Development of Sturm's Form. The condition of monotone increase of $z_{m+1}$ with $z_{m}$ is evidently met by the translation

$$
z_{m+1}=z_{m}+L_{m}
$$

where $L_{m}$ increases monotone with $\lambda\left(L_{m}^{\prime} \geqq 0\right)$. This is a parabolic transformation with its double point at infinity. Each value of $z_{m+1}$ is obtained by a translation of the corresponding value of $z_{m}$ along the real axis in the positive direction. Let now both planes be subjected to the inversion and reflexion,

$$
z_{m+1}=\frac{1}{\bar{z}_{m+1}}, \quad z_{m}=\frac{1}{\bar{z}_{m}} .
$$

The real axis in each plane is preserved, but in the opposite sense, the upper half of the plane being mapped upon the lower half. Hence in the transformed equation,

$$
\bar{z}_{m+1}=\frac{\bar{z}_{m}}{1+L_{m} \bar{z}_{m}}
$$

$\bar{z}_{m+1}$ decreases as $\bar{z}_{m}$ decreases and $L_{m}$ increases. Monotone increase of $\bar{z}_{m+1}$ with $\bar{z}_{m}$ is secured by making the substitution $L_{m}=1 / K_{m}$ and imposing the condition $K_{m}^{\prime} \geqq 0$. The resulting linear relation is

$$
z_{m+1}=\frac{K_{m} z_{m}}{z_{m}+K_{m}}
$$

Let now the $z_{m+1}$ and $z_{m}$ planes be transformed once more, using a linear relation by which merely condition II is preserved. Then in relation (1) the following conditions are sufficient:

$$
\left\{\begin{array}{l}
K_{m} N_{m}-L_{m} M_{m}>0, \\
L_{m}^{\prime} N_{m}-L_{m} N_{m}^{\prime} \leqq 0 .
\end{array}\right.
$$

These conditions are obviously fulfilled in the transformation

$$
\left\{\begin{array}{c}
z_{m}=K_{m} w_{m}-K_{m}, \\
z_{m+1}=K_{m+1} w_{m+1}-K_{m+1},
\end{array}\right.
$$


provided $K_{i}>0, K_{i}^{\prime} \geqq 0,(i=m, m+1)$. Applying this transformation to $(3)$,

$$
K_{m+1} w_{m+1}-K_{m+1}=K_{m}-K_{m} / w_{m} .
$$

This relation has the property that $w_{m+1}$ increases as it vanishes, provided $K_{m}-K_{m} / w_{m}$ increases. Let $w_{m+1}=y_{m+1} / y_{m}, w_{m}=y_{m} / y_{m-1}$, the last equation becomes

$$
K_{m+1}\left(y_{m+1}-y_{m}\right)=K_{m}\left(y_{m}-y_{m-1}\right),
$$

where, if $y_{m}=0, K_{m+1} y_{m+1}=-K_{m} y_{m-1}$, that is, $y_{m+1}$ and $y_{m-1}$ have opposite signs.

If in place of $(3)$ we write

$$
z_{m+1}=\frac{K_{m} z_{m}}{z_{m}+K_{m}}+N_{m}
$$

condition II is still met after the additional translation. Making the same transformation on $z_{m}$ and $z_{m+1}$ as before in obtaining (5), the resulting form is one which will be referred to as STURM's form :

$$
K_{m+1}\left(y_{m+1}-y_{m}\right)=K_{m}\left(y_{m}-y_{m-1}\right)+N_{m} y_{m},
$$

or, using the ordinary difference notation,

$$
\Delta\left(K_{m} \Delta y_{m-1}\right)=N_{m} y_{m} .
$$

If we impose in both (5) and (7), as always, the condition that $y_{0}$ does not vanish throughout the interval, $y_{0}, y_{1} \cdots y_{n}$ form a Sturmian sequence. Here, as in all later similar cases, where the increase of $z_{m+1}$ is conditioned upon the increase of $z_{m}$, the condition of monotone increase must be laid upon $z_{1}$. Thus in this case we must have

$$
z_{1}^{\prime}=\left[\frac{K_{1}\left(y_{1}-y_{0}\right)}{y_{0}}\right]^{\prime} \geqq 0 .
$$

Further for each value of $\lambda$ it is necessary that one or more of the functions upon which conditions of monotone increase are laid shall actually increase. If, for some fixed value of $\lambda,\left[K_{1}\left(y_{1}-y_{0}\right) / y_{0}\right]^{\prime}=0$, we require that for at least one value of $m(m=1,2 \cdots n-1) N_{m}^{\prime}>0$, or that for two consecutive values of $m$, or for $m=n-1, K_{m}^{\prime}>0$.

Development of More General Recurrent Relations. Let the hyperbolic transformation used to secure the first Sturmian condition in (6) be

$$
z_{m}=L_{m} w_{m}-K_{m} \quad\left(L_{m}>0\right),
$$

of which (4) is a special form when $L_{m}=K_{m}$. The resulting recurrent relation is

$$
L_{m+1} y_{m+1}-K_{m+1} y_{m}=\frac{K_{m}}{L_{m}}\left(L_{m} y_{m}-K_{m} y_{m-1}\right)+N_{m} y_{m}
$$




$$
\begin{aligned}
& \Delta\left\{L_{m} \Delta y_{m-1}+\left(L_{m}-K_{m}\right) y_{m-1}\right\} \\
& \quad=\frac{K_{m}-L_{m}}{L_{m}}\left\{L_{m} \Delta y_{m-1}+\left(L_{m}-K_{m}\right) y_{m-1}\right\}+N_{m} y_{m} .
\end{aligned}
$$

Here again both Sturmian conditions are satisfied, provided

$$
L_{m}>0, K_{m}^{\prime} \geqq 0, N_{m}^{\prime} \geqq 0,\left[\frac{L_{1} y_{1}-K_{1} y_{0}}{y_{0}}\right]^{\prime} \geqq 0 .
$$

Instead of beginning with the parabolic substitution (2), we may use the general real elliptic form with fixed points $A_{m}^{(1)}=B_{m}+i C_{m}, A_{m}^{(2)}=B_{m}-i C_{m}$ :

$$
z_{m+1}=\frac{\left(K_{m}+2 B_{m}\right) z_{m}-\left(B_{m}^{2}+C_{m}^{2}\right)}{z_{m}+K_{m}} .
$$

Forming the derivative with regard to $\lambda$, we have

$$
z_{m+1}^{\prime}=\frac{K_{m}^{\prime}\left\{\left(z_{m}-B_{m}\right)^{2}+C_{m}^{2}\right\}+z_{m}^{\prime}\left\{\left(K_{m}+B_{m}\right)^{2}+C_{m}^{2}\right\}}{\left(z_{m}+K_{m}\right)^{2}} .
$$

Here $z_{m+1}$ increases with $\lambda$, provided $K_{m}$ and $z_{m}$ increase. The same transformations already employed upon (5) give the following form :

$$
K_{m+1}\left(y_{m+1}-y_{m}\right)=\left(K_{m}+2 B_{m}\right)\left(y_{m}-y_{m-1}\right)-\frac{B_{m}^{2}+C_{m}^{2}}{K_{m}} y_{m-1}+N_{m} y_{m}
$$

Both Sturmian conditions are satisfied, provided the same conditions are imposed as in (7). It is to be noted that (7) is a special form of both (9) and (11). By writing $C_{m}=0$ in (10) we have the most general parabolic substitution with double point at $B_{m}$. Still another form may be obtained by transforming (10) by means of (8).

The hyperbolic transformation is found to be useful only as an intermediate process in securing the first Sturmian property, under the forms already employed in transforming (6).

In the general linear relation (1) we find that monotone increase of $z_{m+1}$ with $z_{m}$ throughout the interval is secured only by imposing such complicated conditions as make this form too cumbrous for use.

Other recurrent relations may, however, be obtained by combining forms known to satisfy condition II. Consider for example the recurrent relation

$$
z_{m+1}=\frac{\left(K_{m}+2 B_{m}\right) z_{m}-\left(B_{m}^{2}+C_{m}^{2}\right)}{z_{m}+K_{m}}+A_{m} z_{m}+N_{m}
$$

where the following conditions are fulfilled:

$$
K_{m}>0, \quad K_{m}^{\prime} \geqq 0, \quad A_{m} \geqq 0, \quad N_{m}^{\prime} \geqq 0 .
$$

It is evident that $z_{m+1}$ increases monotone with $z_{m}$. Employ now transformation (4), which gives the relation 


$$
\begin{array}{r}
\frac{K_{m+1}\left(y_{m+1}-y_{m}\right)}{y_{m}}=\frac{K_{m}\left(K_{m}+2 B_{m}\right)\left(y_{m}-y_{m-1}\right)-\left(B_{m}^{2}+C_{m}^{2}\right) y_{m-1}}{K_{m} y_{m}} \\
+\frac{A_{m} K_{m}\left(y_{m}-y_{m-1}\right)}{y_{m-1}}+N_{m} .
\end{array}
$$

If $y_{m}=0$, we have

$$
K_{m} K_{m+1} y_{m+1}=-\left\{\left(K_{m}+B_{m}\right)^{2}+C_{m}^{2}\right\} y_{m-1}
$$

and both Sturmian conditions are satisfied.

Other such combinations may readily be made, leading to more or less complicated relations of which (7) will always be found to be a special form. If (12) were transformed by means of (8), the resulting relation would contain both (9) and (13) as special cases.

\section{§4. Properties of a Sturmian Sequence.}

Having thus indicated the method by which the desired recurrent relations are set up, we proceed to a consideration of some of the more important properties of the functions which satisfy these relations.

Graphical Illustration. The variations in the signs of the sequence $y_{0}, y_{1} \cdots y_{n}$ may be illustrated for any fixed value of $\lambda$, as $\lambda_{p}$, by plotting the points whose rectangular coördinates are $x=m, y=y_{m}\left(\lambda_{p}\right),(m=0,1 \cdots n)$. If the consecutive points whose abscissas are $0,1 \cdots n$ be joined, the broken line thus formed cuts the $X$ axis in points which in number and order correspond to the variations in the signs of the functions. These points of intersection will be referred to as the $v$-points of the sequence.

Linear Dependence. Two solutions of a recurrent relation, $y_{m}^{(1)}$ and $y_{m}^{(2)}$, are said to be linearly dependent when two constants $c_{1}$ and $c_{2}$ exist, such that $c_{1} y_{m}^{(1)}+c_{2} y_{m}^{(2)}=0, m=0,1 \cdots n$.

The two following theorems will be needed for later proofs.

Theorem A. Let $y_{m}^{(1)}$ and $y_{m}^{(2)}$ denote two solutions of a linear difference equation of the second order. If the coefficient of the $y$ of highest index does not vanish, the necessary and sufficient condition for the linear dependence of the two solutions is $y_{1}^{(1)} y_{0}^{(2)}-y_{1}^{(2)} y_{0}^{(1)}=0$.

Theorem B. Any solution can be expressed as a linear function of two linearly independent solutions.

The following theorems are stated for the sake of definiteness for a set of functions satisfying the recurrent relation (9), but with certain obvious modifications, often simplifications, they hold for any similar relations.

$$
L_{m+1} y_{m+1}-K_{m+1} y_{m}=\frac{K_{m}}{L_{m}}\left(L_{m} y_{m}-K_{m} y_{m-1}\right)+N_{m} y_{m}
$$


Let the following conditions be satisfied, the equality signs not holding simultaneously for all values of $m$. See p. 427 .

$$
L_{m}>0, K_{m}^{\prime} \geqq 0, N_{m}^{\prime} \geqq 0,\left[\frac{L_{1} y_{1}-K_{1} y_{0}}{y_{0}}\right]^{\prime} \geqq 0 .
$$

Then, according to the previous work, $\left(L_{n} y_{n}-K_{n} y_{n-1}\right) / y_{n-1}$ increases continually with $\lambda$ between two roots of $y_{n-1}, y_{n} / y_{n-1}$ increases when $y_{n}$ vanishes, and $y_{0}, y_{1} \cdots y_{n}$ form a Sturmian sequence.

Theorem I. $A$ variation is lost in the sequence $y_{0}, y_{1} \cdots y_{n}$ whenever $y_{n}$ vanishes.

TheOREM II. The roots of $y_{n}$ and of $y_{n-1}$ separate each other; also the roots of $L_{n} y_{n}-K_{n} y_{n-1}$ and of $y_{n-1}$; also the roots of $L_{n} y_{n}-K_{n} y_{n-1}+M_{n} y_{n-1}$ and of $y_{n-1}$, provided $M_{n}^{\prime} \geqq 0$.

For $\left(L_{n} y_{n}-K_{n} y_{n-1}+M_{n} y_{n-1}\right) / y_{n-1}$ increases continually with $\lambda$ as $\lambda$ passes from one root of $y_{n-1}$ to the next, and assumes in this interval all real values from $-\infty$ to $+\infty$. The numerator therefore vanishes once in every such interval, and, as it can have no multiple roots, only once. If $M_{n}=K_{n}$, the first part of the theorem is proved; if $M_{n}=0$, the second part is proved.

Theorem III. If $y_{m}^{(1)}$ and $y_{m}^{(2)}$ are any two linearly independent solutions of (9) satisfying the condition

$$
\left[\frac{L_{1} y_{1}^{(i)}-K_{1} y_{0}^{(i)}}{y_{0}^{(i)}}\right]^{\prime} \geqq 0
$$

their roots separate each other, provided $K_{m}$ does not vanish throughout the interval considered.

This is proved by showing that $y_{n}^{(1)} y_{n-1}^{(2)}-y_{n}^{(2)} y_{n-1}^{(1)}$ cannot vanish or change sign. On substituting two consecutive roots of one solution this fact is contradicted, unless the other solution changes sign between these two roots.

TheOREM IV. If $L_{m}^{\prime} \geqq 0$, the v-points of the sequence remain stationary or move to the right as $\lambda$ increases.

Let a $v$-point lie between $y_{i}$ and $y_{i-1}$. From the fact of the monotone increase of $L_{m} y_{m}-\dot{K_{m}} y_{m-1} / y_{m-1}$ we have

Hence

$$
\left[\frac{L_{i} y_{i}-K_{i} y_{i-1}}{y_{i-1}}\right]^{\prime} \geqq 0 \text {. }
$$

$$
L_{i}\left(\frac{y_{i}}{y_{i-1}}\right)^{\prime}+L_{i}^{\prime} \frac{y_{i}}{y_{i-1}} \geqq K_{i}^{\prime} \geqq 0 \text {. }
$$

It follows that $\left(y_{i} / y_{i-1}\right)^{\prime} \geqq 0$, and as $\lambda$ increases the $v$-point remains stationary or shifts toward the right.

Theorem V. Let $y_{m}^{(1)}$ and $y_{m}^{(2)}$ be any two linearly independent solutions of (9), and let the only conditions imposed be 


$$
y_{0}^{(1)} \neq 0, \quad y_{0}^{(2)} \neq 0, \quad L_{m} L_{m-1}>0, \quad K_{m} \neq 0 \text {. }
$$

For any given value of $\lambda$ the v-points of the sequences $y_{0}^{(1)}, y_{1}^{(1)} \cdots y_{n}^{(1)}$ and $y_{0}^{(2)}, y_{1}^{(2)} \cdots y_{n}^{(2)}$ separate each other.

Consider the solution $y_{m}=t y_{m}^{(1)}+y_{m}^{(2)}$. By a suitable variation of the parameter $t$ the $v$-points of the sequence $y_{0}, y_{1} \cdots y_{n}$ may be made to move, always in the same sense, from coincidence with the $v$-points of the sequence $y_{0}^{(1)}, y_{1}^{(1)} \cdots y_{n}^{(1)}$ into coincidence with those of the sequence $y_{0}^{(2)}, y_{1}^{(2)} \cdots y_{n}^{(2)}$, without once crossing a $v$-point of either sequence. Hence the $v$-points of the two given sequences separate each other.

Theorem VI. Let $\lambda_{1}$ and $\lambda_{2}$ be any two values of $\lambda\left(\lambda_{1}<\lambda_{2}\right)$, and let $\left(K_{m}^{2} / L_{m}\right)^{\prime} \geqq 0$. Between two consecutive v-points of the sequence $y_{0}\left(\lambda_{1}\right), y_{1}\left(\lambda_{1}\right) \cdots y_{n}\left(\lambda_{1}\right)$ cannot lie more than one v-point of the sequence $y_{0}\left(\lambda_{2}\right), y_{1}\left(\lambda_{2}\right) \cdots y_{n}\left(\lambda_{2}\right)$, and between two consecutive v-points of the latter sequence lies always at least one v-point of the former.

To establish this theorem it may be proved that if a solution has for a given value of $\lambda$, as $\lambda_{1}$, the two consecutive $v$-points $a$ and $b, b$ lying to the right of $a$, a solution may be found which for every value of $\lambda$ has the $v$-point $a$, and that its next $v$-point to the right must lie to the right of $b$. For the value $\lambda=\lambda_{2}$ the $v$-points of this solution and of the given solution must separate each other by Theorem V. The theorem then follows immediately.

Theorem VII. Theorem of Oscillation. If in the interval $\left(\lambda_{i} \lambda_{k}\right) L_{m}$ and $K_{m}$ never exceed in absolute value certain fixed quantities, large at pleasure, if also $N_{m}\left(\lambda_{i}\right) \leqq-P^{2}, N_{m}\left(\lambda_{k}\right) \geqq Q^{2}$, and $P^{2}$ and $Q^{2}$ are sufficiently large, there is one and only one value of $\lambda\left(\lambda_{i} \leqq \lambda \leqq \lambda_{k}\right)$ such that a solution $y_{m}$ exists, satisfying the following conditions:

1. The number of variations in the sequence $y_{1}, y_{2} \cdots y_{n}$ is equal to an assigned integer $r(r<n)$;

2.

$$
\frac{L_{1} y_{1}-K_{1} y_{0}}{y_{0}}=a
$$

3.

$$
\frac{L_{n} y_{n}-K_{n} y_{n-1}}{y_{n-1}}=b
$$

$a$ and $b$ being any real constants.

Using condition 2, $y_{1}$, and therefore all $y$ 's of higher index, can be determined except for the non-vanishing factor $y_{0}$. Owing to the copditions imposed upon the coefficients, there are $n-1$ variations in the sequence $y_{1}, y_{2} \cdots y_{n}$ when $\lambda=\lambda_{i}$ and none when $\lambda=\lambda_{k}$. Since $\left(L_{n} y_{n}-K_{n} y_{n-1}\right) / y_{n-1}$ increases continually with $\lambda$ between two consecutive roots of $y_{n-1}$, condition 3 will be satisfied once and only once in every such interval. 


\section{§5. The Differential Equation.}

A recurrent relation of the second order may be written as an equation in finite differences of the second order, and this, under certain conditions, has as its limiting form a differential equation of the second order. The CAUCHYLipschitz proof of the existence of a solution of a differential equation within a certain interval treats the differential equation as the limit of a difference equation. Painleve and Picard have shown* that the solution converges uniformly to its limit and that the limit represents the solution as long as it remains continuous. It follows that theorems proved for $y_{n}(\lambda)$ in the difference equation can be extended without further proof to $y(x, \lambda)$ in the differential equation which is its limiting form. In particular, theorems proved in $\S 4$ go over at once into their well-known analogues for the differential equation.

Consider for example equation (13), which may be written in the form

$$
\begin{aligned}
y_{m-1} \Delta\left\{K_{m} \Delta y_{m-1}\right\} & -\left\{y_{m-1}\left(2 B_{m}+\frac{B_{m}^{2}+C_{m}^{2}}{K_{m}}+A_{m} K_{m}\right)\right. \\
& \left.+A_{m} K_{m} \Delta y_{m-1}\right\} \Delta y_{m-1}=y_{m} y_{m-1}\left\{N_{m}-\frac{B_{m}^{2}+C_{m}^{2}}{\bar{K}_{m}}\right\} .
\end{aligned}
$$

On making the usual hypotheses in regard to the limits of the functions involved, in order to pass to the differential equation, we find that $A_{m}, B_{m}$ and $C_{m}$ must approach zero, and that different results may be obtained, depending upon the manner of approach. If it is sufficiently rapid, STURM's equation results. If on the other hand $A_{m}, B_{m}$ and $C_{m}$ contain $\delta$ in as low a power as possible ( $\delta$ being one of the equal subdivisions of the $x$ interval, $x=x_{0}+m \delta$ ), other forms are obtained. Thus if we suppose the limits to be the following:

$$
\lim _{\delta=0} \frac{A_{m}}{\delta}=A(x), \quad \lim _{\delta=0} \frac{2 B_{m}}{\delta}=B(x), \quad \lim _{\delta=0} \frac{B_{m}^{2}+C_{m}^{2}}{\delta}=C(x),
$$

from (13)' we have the differential equation

$$
\frac{d}{d x}\left(K \frac{d y}{d x}\right)-\left(B+\frac{C}{K}+A K\right) \frac{d y}{d x}=N y \text {. }
$$

This possesses the same general properties as STuRM's equation, providing the following conditions are met :

$$
K>0, K^{\prime} \geqq 0, A \geqq 0 . C \geqq 0, N^{\prime} \geqq 0,\left(\frac{K \frac{d y}{d x}}{y}\right)_{x=x_{0}}^{\prime} \geqq 0, \dagger
$$

not all the signs of equality holding simultaneously.

* Bulletin de la Société Mathématique de France, vol. 27 (1899), p. 150. Comptes Rendus, vol. 128 (1899), p. 1363.

$\dagger$ The sign of differentiation in each case refers as before to $\lambda$. 
From (14) six special forms may be derived by letting one or two of the coefficients $A, B, C$, vanish.

Consider further equation $(9)^{\prime}$, which may be simplified by writing $L_{m}-K_{m}=M_{m}$, whence $L_{m}^{\prime} \geqq M_{m}^{\prime}$. This leads directly to the differential equation

$$
\frac{d}{d x}\left(L \frac{d y}{d x}+M y\right)=\frac{M}{L}\left(L \frac{d y}{d x}+M y\right)+N y,
$$

with the conditions

$$
L>0, L^{\prime} \geqq M^{\prime}, N^{\prime} \geqq 0, \quad\left[\frac{L \frac{d y}{d x}+M y}{y}\right]_{x=x_{0}}^{\prime} \geqq 0,
$$

not all the signs of equality holding simultaneously.

The theorems proved above for equation (9) are evidently immediately applicable to equation (15).

It may readily be shown that each of the equations (14) and (15), together with the six special forms referred to, is more general than the equation discussed by STURM. For, by the transformation $y=u v$ and by removal of the term in $d v / d x$, each may be reduced to the general type to which Sturm's equation belongs:

$$
\frac{d}{d x}\left(R \frac{d v}{d x}\right)=S v
$$

But in every case it is apparent that the conditions laid upon the function $S$ do not ensure its monotone increase with $\lambda$, while in the case of (15) the same statement holds true for $R$. It follows that not simply equations (14) and (15), but many specialized forms of the former as well, are more general than the equation of STuRM, which appears as a special case under each.

Hence the properties proved in STURM's memoir for a special equation are seen to belong to a more extensive class of equations, which are derived by most simple methods, and which clearly all possess an oscillation theorem in the Sturmian sense.

YALE UNIVERSITY, May, 1903. 